Conclusions: The introduction of acute sedation practice guidelines in the Alfred in-patient psychiatry service improved staff safety by decreasing the number of staff injuries and improved patient outcomes by decreasing the number of patient falls and patient-onpatient assaults.

\section{Wagging the black dog: predicting depression severity using neuropsychological measures}

\section{P Hopkinson 1,2, A Kemp' ${ }^{1}$, E Gordon², R Clarke ${ }^{3}$, P Boyce $^{4}$, L Williams ${ }^{1}$}

'Brain Dynamics Centre; 'Brain Resource Company; ${ }^{3}$ Flinders University of South Australia; and ${ }^{P}$ sychological Medicine, Westmead Hospital, Australia

Background: Depression poses an enormous burden on both the individual and the community. However, relatively little is known about the mechanisms that underpin the disorder. Core neuropsychological domains include memory, executive, sensorimotor, attention and verbal functions. However, the conceptualization of depression usually involves the implementation of discrete variables. We decided to integrate these core neuropsychological domains to predict depression severity.

Methods: Fifty patients clinically diagnosed with major depressive disorder and 200 age- and sex-matched controls undertook a neuropsychological test battery. A regression analysis was carried out to predict depression severity, as indexed by scores on the Hamilton Rating Scale for Depression-17 and Depression, Anxiety and Stress Scales.

Results: Preliminary regression analyses show that an integration of neuropsychological indexes from the core domains predicted depression severity. Statistically significant interactions between these variables also predicted depression severity.

Conclusions: We showed that integrating theoretically relevant neuropsychological variables such as sensorimotor and verbal functions provided valuable insight into the understanding and prediction of depression severity. These findings offer insight into the endophenotypic nature of major depressive disorder. Future studies could implement similar methodology for the prediction of treatment response in depression.

\section{The mental health and well-being of rural people: a pilot study measuring community factors}

\author{
F Hourihan, T Lewin, H Stain, B Kelly \\ Centre for Rural and Remote Mental Health, University of Newcastle, Orange, \\ Australia
}

Background: This study was a pilot for an NHMRCfunded project examining mental health and well-being among 4000 rural households in New South Wales. Aim: To investigate relationships between novel indexes detailing perceived features of the rural community and psychological distress.

Methods: A survey was mailed to 2000 adults (aged 18 years or older) randomly selected through the electoral roll from four local government areas of varying remoteness. Perceived community support (infrastructure and support networks) and community attachment were measured using items from a 'Community Participation Survey' and 'Feelings about Living in the Community' (Higginbotham et al. 2005). Postulated rural stressors included drought impact and access to health care. Psychological distress was measured using the Kessler-10. Results: The sample $(n=449$; response rate $24 \%)$ was slightly overrepresented by women $(58 \%)$ and older age groups (mean 51 years \pm 15 ) and $29 \%$ were farmers/farmworkers. Moderate to very high psychological distress $(\mathrm{K} 10>15)$ was reported in $23 \%$ of the sample. 'Worry/stress' from the drought was reported by $57 \%$ of respondents. Psychological distress also correlated with 'worry/stress' from rural stressors. In a hierarchical regression analysis, neuroticism, perceived health, number of stressful life events and perceived community support and attachment explained $52 \%$ of the variance in psychological distress.

Conclusions: This pilot study has highlighted an association between perceived features of the rural community and mental health, which will be explored in greater detail in our ongoing research investigating determinants of mental health and well-being within rural communities.

\section{Adolescent alcohol use and mobile phone experience sampling in a clinical setting: an innovative, youth friendly approach to research}

\author{
S Kauer ${ }^{1,2}$, S Reid', L Sanci ${ }^{3}$, G Patton' \\ ${ }^{1}$ Centre for Adolescent Health, Murdoch Children's Research Institute; \\ ${ }^{2}$ Royal Childrens Hospital; and ${ }^{3}$ The University of Melbourne, Melbourne, Australia
}

Background: Adolescent alcohol use is a continuing problem probably influenced by many motivational factors. Capturing the daily experiences of young people may increase understanding of these factors and momentary sampling provides a possible means to do so. The aim of this study was to develop a mobile phone momentary sampling program to monitor the daily experiences of young people who drink alcohol at high-risk levels and track their moods, stresses and activities across each day. A concurrent aim was to pilot the program in a clinical setting. 